\title{
Prevalence of headache in Australian footballers
}

\section{P McCrory, J Heywood, C Coffey}

Br J Sports Med 2005;39:e10 (http://www.bjsportmed.com/cgi/content/full/39/2/e10). doi: 10.1136/bjsm.2004.014860

\begin{abstract}
Objective: To survey the prevalence and risk factors for headache in a population of elite professional Australian footballers.

Methods: A prospective questionnaire based survey was performed on elite Australian footballers participating in a national competition. The survey was designed to assess the prevalence and risk factors for headache using standardised International Headache Society (HIS) criteria. Headache prevalence was compared with that of an age and sex matched community control population.

Results: A total of 160 questionnaires were analysed. Headaches were reported by $80 \%$ of subjects, with $49 \%$ of respondents reporting headaches during competitive match play and $60 \%$ during training. There was no significant difference between the footballers and the community sample in the number of headaches ever; however, they did report more headaches in the three months before the survey - that is, during the competitive playing season. In the footballers, $22 \%$ of headaches conformed to the strict IHS definition of migraine headaches. When the relaxed definition of "footballer's migraine" was used, 34\% of headaches met these criteria. Footballers were at significantly increased risk of footballer's migraine than community controls.

Conclusions: Headaches are common in Australian footballers, and the prevalence of migraine is increased in relation to community prevalence studies. Furthermore, the strict IHS criteria may not adequately identify the specific subtype of football related headache. This finding has important implications in the management of headache in this setting.
\end{abstract}

A lthough community studies of headache note exercise as a potent trigger of migraine and other headache syndromes, the prevalence and nature of headache in sport is largely unknown. ${ }^{1}$

The only published study on sport related headaches is a clinic based retrospective survey performed on university students participating in varsity sport. The authors found that headaches were reported by $35 \%$ of all respondents, with no sex effect evident. ${ }^{23}$ There have also been anecdotal case reports of migraine and other headache syndromes occurring in a variety of sports. ${ }^{4-6}$

In this study, using standardised International Headache Society (IHS) diagnostic criteria, ${ }^{7}$ we prospectively surveyed the prevalence and risk factors for headache in a population of elite professional Australian footballers and compared the headache prevalence with that of a non-sporting community population. ${ }^{8}$

\section{METHODS}

Study participants were randomly selected from registered players in the Australian Football League by a two stage sampling procedure. Firstly, eight teams were randomly selected from the 16 teams in the league. Secondly, 20 players were selected randomly from each team. After giving written informed consent, participants completed a self administered anonymous questionnaire. The survey was conducted at the midpoint of the competitive playing season. The survey instrument was based on the questionnaire used in a concurrent prevalence study of headache conducted in Victoria, Australia. ${ }^{8}$ Additional questions were included to characterise the sport participation in more detail and, in particular, to identify specific types of exercise that may provoke headache.

Headaches were classified as migraine and non-migraine headache according to IHS criteria. ${ }^{7}$ The IHS criteria represent the standardised international diagnostic criteria for all headache syndromes applicable to research studies. A number of headaches met all IHS criteria for migraine except the criterion that specified headache duration of 4-72 hours. In order not to exclude potential migraine variants, a separate category of "footballer's migraine" was created in which all IHS criteria were met but the minimum duration criterion of four hours was relaxed, thereby including headaches of shorter duration.

The results were compared with a community sample matched for sex and age. The community sample was derived from a concurrently performed community headache prevalence survey using a telephone based questionnaire sampling method. ${ }^{9}$

\section{Statistical analysis}

Data analysis was carried out using the STATA program (version 6.0; 1999; STATA Corporation, Houston, Texas, USA). STATA's "survey estimation" command (svymean) with football team set as the strata variable was used in the estimation of prevalence rates to allow the cluster sampling procedure. Multivariate logistic regression was used to compare the footballer sample with the community sample with adjustment for age. All confidence intervals are reported at the $95 \%$ level of significance.

\section{RESULTS}

A total of 160 questionnaires were obtained from eight teams giving a $100 \%$ response rate. This sample constituted $24 \%$ of the total number of registered professional players in this football code. The mean (SD) age of the respondents was 25.0 (5.2) years (range 17-35) and all were male.

Headaches were reported by $125 / 160(78 \%)$ subjects, with $49 \%$ of respondents having headaches during competitive match play and $60 \%$ during training. All headaches in this study were clinically benign, and no catastrophic brain injuries were observed during the time frame of the study.

The prevalence of headaches in footballers was compared with that of the community sample. There was no significant difference between the footballers and the community sample in the number of headaches ever (odds ratio (OR) $0.64,95 \%$ confidence interval (CI) 0.37 to 1.1 ). However, they did report more headaches in the three months before the survey - that is, the duration of the playing season (OR 6.3, 95\% CI 2.7 to 14.7 ). 
Table 1 Prevalence of International Headache Society (HIS) defined migraine and "footballer's migraine" by activity

\begin{tabular}{|c|c|c|c|c|}
\hline \multirow[b]{2}{*}{ Activity } & \multicolumn{2}{|c|}{ IHS criteria migraine } & \multicolumn{2}{|c|}{ Footballer's migraine } \\
\hline & $\%$ & $95 \% \mathrm{Cl}$ & $\%$ & $95 \% \mathrm{Cl}$ \\
\hline Hit to head & 1.3 & 0 to 3.0 & 5.0 & 1.6 to 8.4 \\
\hline Running & 0 & 0 & 2.5 & 0.1 to 4.9 \\
\hline Weight training & 0.6 & 0 to 1.9 & 0.6 & 0 to 1.9 \\
\hline Any football activity* & 2.5 & 0 to 5.0 & 10.0 & 5.2 to 14.8 \\
\hline Unrelated to football & 2.5 & 0 to 5.0 & 2.5 & 0.1 to 4.9 \\
\hline Combined symptoms over all activities $\dagger$ & 4.4 & 1.1 to 7.6 & 13.8 & 8.3 to 19.2 \\
\hline
\end{tabular}

Values are percentages; $N=160$. Note: the categories are not mutually exclusive.

*Symptoms from all football related activities were pooled to generate IHS or footballer's migraine diagnosis.

†Symptoms from all activities were pooled to generate IHS or footballer's migraine diagnosis.

In the footballers, 28/125 headache respondents conformed to the strict IHS definition of migraine headaches. When the relaxed definition of "footballer's migraine" was used, 43/125 headaches met these criteria. The community sample was then assessed using the footballer's migraine criteria. Footballers were at increased risk of footballer's migraine compared with community controls (OR 3.5, 95\% CI 2.0 to $6.3)$.

Table 1 displays prevalence of football migraine by activity. The IHS migraine and footballer's migraine criteria were assessed separately for each football activity (hit to the head, training, running, etc). Symptoms were then pooled across these football related activities to generate a "football related" diagnosis. Similarly, symptoms were pooled across all activities including non-football activities to generate an overall diagnosis. The prevalence of IHS migraine in this group of footballers was no greater than zero for any football related or unrelated activity. When criteria were pooled, $4 \%$ met the strict IHS migraine criteria. When the duration criterion was relaxed, $10 \%$ of footballers reported footballer's migraine related to football activities in general, with the most contribution coming from hits to the head. A total of $14 \%$ reported footballer's migraine assessed from pooled information.

\section{DISCUSSION}

Previously published epidemiological data suggest that sport related headache is common, although the precise nature of the headaches and epidemiology remains unclear. ${ }^{23}$ One would intuitively expect that, in a collision sport, such as Australian football, where body and head impact is common, the prevalence of headaches would be high. In this study, we show that about $80 \%$ of the players report headache. Of these, $22 \%$ conform to the strict IHS criteria for migraine.

A much larger group (34\%), however, reports migrainous headaches that do not conform to all of the strict IHS criteria. In this group, the major limitation of the diagnostic criteria is the duration of the headache. Approximately one third of all headaches last less than four hours, and this may represent a subset of trauma triggered migraine which requires different criteria from those used in community studies.
In this study, no attempt was made to distinguish headaches that may be triggered from the cervical spine (cervicogenic headache). Such syndromes cannot be reliably diagnosed by questionnaire alone.

The accurate diagnosis of headache syndromes in sport has important treatment implications. ${ }^{19}$ In professional sport, many conventional headache medications (such as $\beta$ adrenergic antagonists, caffeine, codeine-containing preparations, dextropropoxyphene, narcotics, opioids, etc) are banned under International Olympic Commission rules. Accordingly the ability of a team doctor to accurately diagnose and treat the specific headache requires an understanding of the symptomatology and nature of headaches that may present in these situations.

\section{Authors' affiliations}

P McCrory, J Heywood, C Coffey, CHESM/School of Physiotherapy, University of Melbourne, Victoria, Australia

Competing interests: none declared

Correspondence to: Dr Paul McCrory, CHESM/School of Physiotherapy, University of Melbourne, Melbourne, Victoria 3010, Australia; paulmccr@bigpond.net.au

Accepted 8 August 2004

\section{REFERENCES}

1 McCrory P. Headaches and exercise. Sports Med 2000;30:221-9.

2 Williams S, Nukada H. Sport and exercise headache. Part 2. Diagnosis and classification. Br J Sports Med 1994;28:96-100

3 Williams S, Nukada H. Sport and exercise headache. Part 1. Prevalence amongst university students. Br J Sports Med 1994;28:90-5.

4 Gibbs N. Common rugby league injuries. Recommendations for treatment and preventative measures. Sports Med 1994;18:438-50.

5 Matthews WB. Footballer's migraine. BMJ 1972;2:326-7

6 Rasmussen B. Epidemiology of headache. Cephalgia 1995;15:45-68

7 International Headache Society. Headache Classification Committee of the International Headache Society: classification and diagnostic criteria for headache disorders, cranial neuralgias and facial pain. Cephalgia 1997; 18(suppl):43-110.

8 Heywood J, Colgan T, Coffey C. Prevalence of headache and migraine in an Australian city [abstract]. J Clin Neurosci 1998:5:485.

9 McCrory P. Headache in sport. In: Brukner P, Khan K, eds. Clinical sports medicine. Sydney: McGraw-Hill, 1993. 